\title{
Investigating the rp-process with the Canadian Penning trap mass spectrometer
} \author{
J.C. Hardy ${ }^{5}$, A.A. Hecht ${ }^{1,6}$, A. Heinz ${ }^{1}$, J.K.P. Lee ${ }^{4}$, A.F. Levand ${ }^{1}$, B.F. Lundgren ${ }^{1}$, R.B. Moore ${ }^{4}$, G. Savard ${ }^{1}$, \\ N.D. Scielzo ${ }^{1}$, D. Seweryniak ${ }^{1}$, K.S. Sharma ${ }^{2}$, G.D. Sprouse ${ }^{7}$, W. Trimble ${ }^{1}$, J. Vaz ${ }^{1,2}$, J.C. Wang ${ }^{1,2}$, Y. Wang ${ }^{1,2}$, \\ B.J. Zabransky ${ }^{1}$, and Z. Zhou ${ }^{1}$ \\ 1 Physics Division, Argonne National Laboratory, Argonne, IL 60439, USA \\ 2 Department of Physics and Astronomy, University of Manitoba, Winnipeg, MB R3T 2N2, Canada \\ 3 Centre d'Etudes Nucléaires de Bordeaux-Gradignan, F-33175 Gradignan Cedex, France \\ 4 Department of Physics, McGill University, Montreal, QC H3A 2T8, Canada \\ 5 Cyclotron Institute, Texas A\&M University, College Station, TX 77843-3366, USA \\ 6 Department of Chemistry, University of Maryland, College Park, MD 20742, USA \\ 7 Physics Department, SUNY, Stony Brook University, Stony Brook, NY 11794, USA
}

J.A. Clark ${ }^{1,2, a}$, R.C. Barber ${ }^{2}$, B. Blank ${ }^{1,3}$, C. Boudreau ${ }^{1,4}$, F. Buchinger ${ }^{4}$, J.E. Crawford ${ }^{4}$, J.P. Greene ${ }^{1}$, S. Gulick ${ }^{4}$,

Received: 15 January 2005 /

Published online: 15 August 2005 - (C) Società Italiana di Fisica / Springer-Verlag 2005

\begin{abstract}
The Canadian Penning trap (CPT) mass spectrometer at the Argonne National Laboratory makes precise mass measurements of nuclides with short half-lives. Since the previous ENAM conference, many significant modifications to the apparatus were implemented to improve both the precision and efficiency of measurement, and now more than 60 radioactive isotopes have been measured with half-lives as short as one second and with a precision $(\Delta m / m)$ approaching $10^{-8}$. The CPT mass measurement program has concentrated so far on nuclides of importance to astrophysics. In particular, measurements have been obtained of isotopes along the rp-process path, in which energy is released from a series of rapid proton-capture reactions. An X-ray burst is one possible site for the rp-process mechanism which involves the accretion of hydrogen and helium from one star onto the surface of its neutron star binary companion. Mass measurements are required as key inputs to network calculations used to describe the rp-process in terms of the abundances of the nuclides produced, the light-curve profile of the X-ray bursts, and the energy produced. This paper will present the precise mass measurements made along the rp-process path with particular emphasis on the "waiting-point" nuclides ${ }^{68} \mathrm{Se}$ and ${ }^{64} \mathrm{Ge}$.
\end{abstract}

PACS. 21.10.Dr Binding energies and masses - 26.30.+k Nucleosynthesis in novae, supernovae, and other explosive environments $-26.50 .+\mathrm{x}$ Nuclear physics aspects of novae, supernovae, and other explosive environments

\section{Introduction}

Since the previous ENAM conference [1], the masses of more than 60 radioactive isotopes have been determined with the Canadian Penning trap (CPT) mass spectrometer at Argonne National Laboratory (ANL) [2,3,4,5]. Most of the isotopes studied were selected due to their important role in astrophysical processes such as the rpprocess [6] discussed here.

A possible site for the rp-process is a binary star system in which hydrogen and helium from a gas giant accrete onto the surface of its neutron star companion. As material accumulates onto the surface, the gravitational pres-

\footnotetext{
${ }^{\mathrm{a}}$ Conference presenter;

e-mail: jclark@physics.umanitoba.ca
}

sure can become sufficient to ignite nuclear burning of the hydrogen and helium [7]. With accretion rates of $\sim 10^{-9}$ solar masses per year, the continuing increase in pressure (and temperature) eventually results in a thermonuclear runaway [8]. At this time, the creation of heavier elements is realized through a series of rapid proton-capture reactions. The resulting energy release is ultimately observed as an X-ray burst [9] with a typical duration of 10-100 seconds.

During the thermonuclear runaway, the reaction path is set by thousands of reaction rates, including those of predominant proton-capture, photodisintegration, and $\beta$-decay reactions. Nuclides along this path, where the proton-capture reactions are hindered by photodisintegration reactions, are termed "waiting-point" nuclides. At 
these nuclides, the rp-process stalls and cannot continue until the destruction of the waiting-point nuclide, either through its $\beta$ decay, or through the rapid capture of two protons to bypass the impeding nucleus. The timescale of the rp-process, or equivalently the light-curve profile of X-ray bursts, depends largely upon the contribution of the individual delays at each waiting-point nuclide. Since these delays are dependent upon the reaction rates of the nuclides involved, information about $\beta$-decay half-lives and energy levels which can be thermally populated are necessary to assess the delay at each waiting-point nuclide. Furthermore, since the photodisintegration rates are exponentially dependent upon proton separation energies [7], masses of waiting-point nuclides are critical. Even a small uncertainty in the masses yields a large uncertainty in the effective half-lives of, or delays at, the waiting-point nuclides.

The potential waiting-point nuclides are the even $N=Z$ nuclei due to the predicted structure of the proton drip-line. Since the delay each waiting-point nuclide contributes to the timescale of the rp-process is at most equal to its $\beta$-decay lifetime, the waiting-point nuclides which could have the biggest influence are those at the beginning of the $N=Z$ chain, in particular ${ }^{64} \mathrm{Ge}$ and ${ }^{68} \mathrm{Se}[7]$.

\section{Measurements}

Precise mass measurements made with the CPT require sufficient yields of low-energy ions with minimal contamination. Since the objective of the CPT is to make measurements of short-lived, rare isotopes, the injection system must be efficient and quickly prepare the ion samples. As a description of the CPT apparatus has been given previously $[10,11]$, only a brief description is provided here.

Nuclides along the rp-process path were produced from fusion-evaporation reactions between heavy-ion beams from the ATLAS facility at ANL and a rotating target wheel. From there, the high energy nuclides of interest are collected and focussed into a gas cell [12] where they are thermalized with 200 mbar of purified helium gas. Within milliseconds, the nuclides are extracted from the gas catcher, proceed through a differentially pumped ion cooler [13], and enter an isotope separator [14] where contamination is reduced. The ions are then transferred to a linear radio-frequency quadrupole trap (RFQ trap) where they are cooled with helium buffer gas and accumulated. Once the precision Penning trap is ready for more ions, the prepared bunch of pure, low-energy ions in the RFQ are transferred to the precision Penning trap. The efficiency of the entire system, from production to detection, is on the order of $0.1 \%$. Ions can be delivered to the precision Penning trap within 100 milliseconds and with an energy spread of $\sim 1 \mathrm{eV}$.

Ions are confined in the Penning trap with a superposition of a magnetic and electric field [15]. Radial confinement is provided by a $5.9 \mathrm{~T}$ homogeneous magnetic field and axial confinement is achieved by a harmonic potential created by applying appropriate voltages on the electrode structure of the Penning trap. The central, or ring, electrode is divided into quadrants enabling the application of an azimuthally symmetric time-varying quadrupole field. In this manner $[16,17]$, the trapped ions can be driven resonantly at their cyclotron frequency:

$$
\omega_{c}=\frac{q e B}{m}
$$

where the charge of the electron is indicated by $e$. Since the cyclotron frequency depends only upon the magnetic field strength $B$, the charge state $q$, and the mass $m$ of the ion, accurate and precise mass measurements can be made. Furthermore, from eq. (1) and ref. [18], the precision in the mass measurement can be shown to follow the relation

$$
\frac{\delta m}{m}=\frac{\delta \omega_{c}}{\omega_{c}} \propto \frac{m}{q e B} \frac{1}{t_{R F} \sqrt{N}},
$$

with $N$ representing the total number of ions detected and $t_{R F}$ is the duration of the applied quadrupole field. Therefore, under similar operating conditions, higher precision is naturally obtained for lighter masses.

The effect of the excitation is determined by a time-offlight (TOF) method [19] in which a multichannel scaler records the time it takes for the ions to reach a microchannel plate detector (MCP) after ejection from the Penning trap. Radial energy gained from the excitation is converted into axial energy by the interaction of the magnetic moment of the ions with the magnetic field gradient outside the Penning trap. If the trapped ions were resonantly excited at their cyclotron frequency, they reach the detector sooner than ions not driven at their cyclotron frequency. This technique is a destructive one; each measurement cycle requires a new ion bunch. To determine the resonant cyclotron frequency, each ion bunch is subjected to a slightly different excitation frequency. After plotting the average arrival time of the ions at the MCP detector as a function of the excitation frequency, the cyclotron frequency is obtained by determining the minimum in the spectrum.

Determining the strength of the magnetic field is achieved by periodically measuring the cyclotron frequency of a well-known mass, $m_{\text {ref }}$. Assuming this reference mass and the unknown mass are subjected to the same magnetic field, the mass of the unknown nuclide is calculated by

$$
m=\left(\frac{q}{q_{\mathrm{ref}}}\right)\left(\frac{\omega_{c, \mathrm{ref}}}{\omega_{c}}\right)\left(m_{\mathrm{ref}}-q_{\mathrm{ref}} m_{e}\right)+q m_{e},
$$

where $m_{e}$ represents the mass of the electron.

\section{Results}

The plotted curve in fig. 1 shows how the effective half-life of ${ }^{68} \mathrm{Se}, t_{1 / 2 \text {,eff }}$, depends upon the proton-capture $Q$ value, $Q_{p}$, for typical conditions during an X-ray burst [2]. For small $Q_{p}$ values, the proton-capture rate is small in comparison with the photodisintegration rate of ${ }^{69} \mathrm{Br}$, and so 


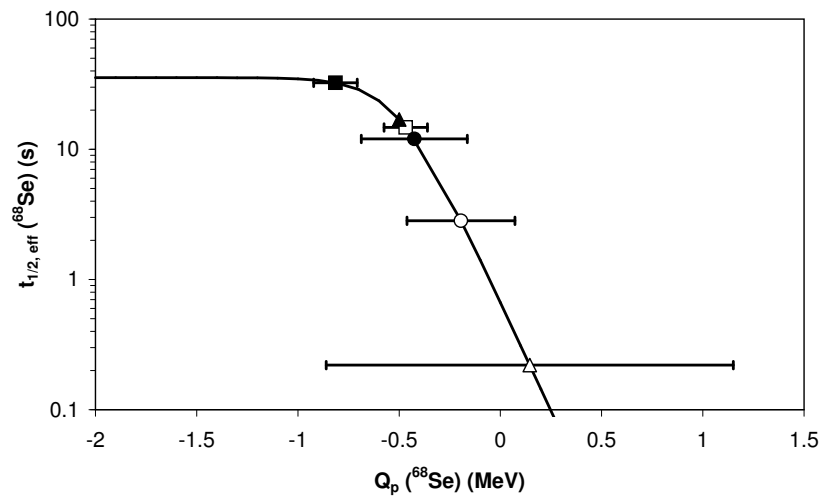

口CPT - AME $\triangle$ SPEG - AME o CSS2 - AME • FMA - AME — CPT - HF \ Pfaff et al.

Fig. 1. The effective half-life of ${ }^{68} \mathrm{Se}$ as a function of the proton-capture $Q$ value for ${ }^{68} \mathrm{Se}$. For the data points with a two-name legend entry, the first name indicates the source of the ${ }^{68}$ Se mass (SPEG [20], CSS2 [21], or FMA [22]) and the second name represents the source of the ${ }^{69} \mathrm{Br}$ mass (AME [23] or $\mathrm{HF}[24])$. Also shown is the upper limit for $Q_{p}\left({ }^{68} \mathrm{Se}\right)$ as determined by Pfaff et al. [25]. The curve demonstrates how the effective half-life of ${ }^{68}$ Se depends upon the proton-capture $Q$ value and is not a fit to the data. It was calculated under conditions typical for X-ray burst models.

the rp-process is stalled until the $\beta$ decay of ${ }^{68} \mathrm{Se}$ occurs. For larger $Q_{p}$ values, the increased likelihood of destruction of ${ }^{68}$ Se via proton-capture decreases the effective halflife of ${ }^{68} \mathrm{Se}$ since the rp-process can continue before the $\beta$ decay of ${ }^{68}$ Se takes place.

Along the plotted curve in fig. 1 are data points which represent the $Q_{p}$ values determined by various groups. Since $Q_{p}\left({ }^{68} \mathrm{Se}\right)$ requires both the mass of ${ }^{68} \mathrm{Se}$ and that of ${ }^{69} \mathrm{Br}$, the first name in each legend entry indicates the source of the ${ }^{68} \mathrm{Se}$ mass, and the second name represents the source of the ${ }^{69} \mathrm{Br}$ mass. As ${ }^{69} \mathrm{Br}$ is proton unbound, its short-lived nature prevents a precise measurement of its mass. Instead, its mass is better estimated by HartreeFock (HF) calculations [24]. Now if our mass determination of ${ }^{68} \mathrm{Se}[2]$ is used in conjunction with $\mathrm{HF}$ calculations for the mass of ${ }^{69} \mathrm{Br}$, the effective half-life of ${ }^{68} \mathrm{Se}$ is between 29 and $34 \mathrm{~s}$, and therefore ${ }^{68}$ Se provides a significant delay during the rp-process.

As ${ }^{64} \mathrm{Ge}$ has a $\beta$-decay half-life of $64 \mathrm{~s}$, its effective halflife could potentially contribute a larger portion to the total timescale of the rp-process. If we combine our mass determination of ${ }^{64} \mathrm{Ge}$ with the Hartree-Fock calculations for ${ }^{65} \mathrm{As}$ [24], the effective half-life of ${ }^{64} \mathrm{Ge}$ is then between 0.7 and $5.7 \mathrm{~s}$. This would indicate ${ }^{64} \mathrm{Ge}$ is a waiting-point nuclide, but not as significant as ${ }^{68} \mathrm{Se}$.

The masses of nuclides along the rp-process path, especially in the vicinity of the proton-rich $\mathrm{Ru}, \mathrm{Rh}$, and Pd nuclides, have not been measured precisely, if at all. Our analysis to date [26] has resulted in the mass determinations of 10 nuclides in this region. Our preliminary results are shown in fig. 2. Here, the differences between our measurements and the mass values from the 2003 atomic mass evaluation (AME) [23] are shown with

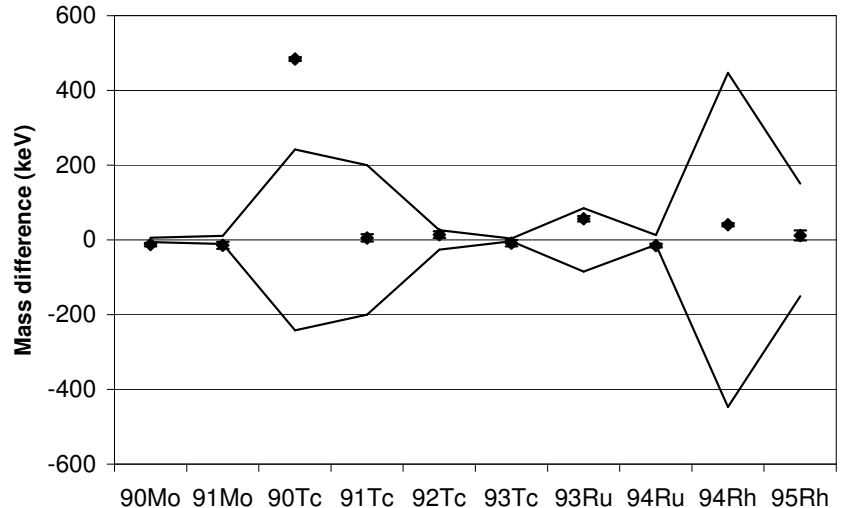

Fig. 2. Mass measurements of proton-rich refractory metals performed by the CPT. Plotted are the differences between the preliminary CPT results and those from AME. The error bars shown represent only the statistical uncertainty from the CPT results ( $8 \mathrm{keV}$ on average), with the lines indicating the uncertainty in the masses from AME. In two cases, ${ }^{90} \mathrm{Tc}$ and ${ }^{91} \mathrm{Tc}$, it is uncertain as to whether the ground or isomeric state was measured.

the lines indicating the precision of the masses as quoted in AME. Our measurements are plotted with statistical error bars only. Excellent agreement exists between our measurements and those in AME, but our results were obtained with much better precision. For two nuclides, ${ }^{90} \mathrm{Tc}$ and ${ }^{91} \mathrm{Tc}$, our measurements were performed with resolution insufficient to resolve the isomers (with excitation energies of $310(390) \mathrm{keV}$ and $139.3(0.3) \mathrm{keV}$ respectively [23]) from the ground state, and so it is possible our TOF spectra have a mixture of both. We intend to revisit this region to measure more proton-rich nuclides closer to the rp-process path with an increased resolving power to discern the ground and isomeric states.

The rp-process path is thought to terminate in the $\mathrm{Sb}, \mathrm{Sn}$, and Te region [27]. The Te isotopes are known ground-state alpha emitters, so a cycle is established between the proton capture reactions into the Te isotopes and the subsequent alpha emission of these isotopes. Uncertainties in the masses of the nuclides in this region ultimately affect the resulting nuclide abundances. The preliminary results [26] of our data for 10 nuclides in this region are shown in fig. 3 where again the differences between our measurements and the AME values are plotted. The lines show the uncertainties in the masses from AME. Our measurements, with much better precision, mostly agree with AME. For two cases, ${ }^{104}$ In and ${ }^{106}$ In, we could not resolve the ground and known isomeric states (with excitation energies $93.48(0.10) \mathrm{keV}$ and $28.6(0.3) \mathrm{keV}$, respectively [23]). We plan to revisit this region to resolve this issue.

Mass measurements are also critical for the study of novae, which are similar to X-ray bursts with the neutron star substituted by a white dwarf. Novae outbursts lead to the production of ${ }^{22} \mathrm{Na}$ which can be used as an observable of these events [28] by detecting the emission of the $1.275 \mathrm{MeV} \gamma$-ray following its $\beta$ decay. However, one of the main uncertainties in the production of ${ }^{22} \mathrm{Na}$ is the 


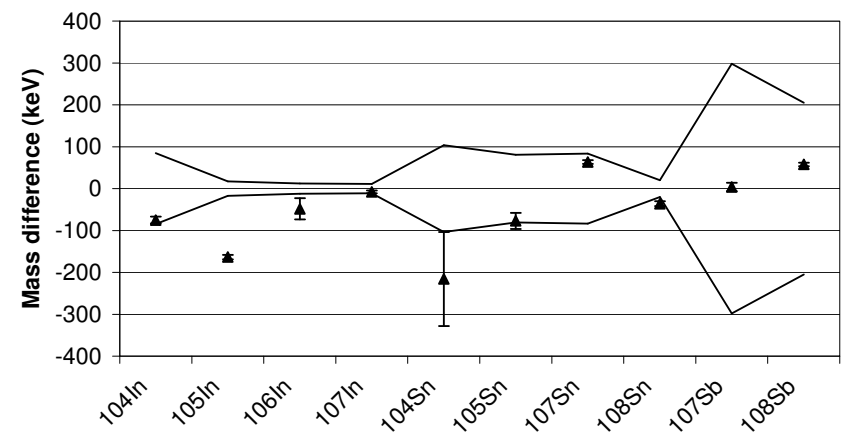

Fig. 3. Mass measurements of nuclides near the proposed endpoint of the rp-process. Plotted are the differences between the preliminary CPT results and those from AME. The error bars shown represent only the statistical uncertainty from the CPT results (20 keV on average), with the lines indicating the uncertainty in the masses from AME. In two cases, ${ }^{104} \mathrm{In}$ and ${ }^{106} \mathrm{In}$, it is uncertain as to whether the ground or isomeric state was measured.

${ }^{21} \mathrm{Na}(\mathrm{p}, \gamma){ }^{22} \mathrm{Mg}$ reaction rate [29] which leads to the importance of the ${ }^{22} \mathrm{Mg}$ mass as ${ }^{21} \mathrm{Na}$ is already well known [23]. The current mass evaluation (AME) based the mass of ${ }^{22} \mathrm{Mg}$ on two measurements published in $1974[30,31]$. However, a recent experiment at ISAC at TRIUMF [32], which directly measured the energy of the resonance into the astrophysically relevant $2^{+}$state, suggested that the mass excess of ${ }^{22} \mathrm{Mg}$ should shift down by about $6 \mathrm{keV}$ compared to AME assuming the energy of the $2^{+}$state was correct. Since then, the mass excess of one of the initial mass measurements [30] has been lowered by $3 \mathrm{keV}$ to account for the change in the ${ }^{16} \mathrm{O}(p, t)^{14} \mathrm{O} Q$ value [33] tied to the original measurement [34]. Our precise mass measurement [3], in agreement with a recent measurement conducted by ISOLTRAP [35], only accounts for $3 \mathrm{keV}$ of the $6 \mathrm{keV}$ shift suggested by ref. [32]. The remaining 3 $\mathrm{keV}$ discrepancy was removed following a more accurate measurement of the $2^{+}$energy level [36]. All the recent measurements now yield consistent results.

With respect to tests of the unitarity of the CKM matrix, our mass of ${ }^{22} \mathrm{Mg}$ provides for a corrected $\mathrm{Ft}$ value of $3081(8) \mathrm{s}$, with the uncertainty now dominated by the branching ratio.

\section{Summary}

Mass measurements are required to characterize X-ray bursts in terms of the abundance of the elements produced, the energy released, and the timescale of the X-ray burst. The timescale is influenced heavily by the individual delays of the waiting-point nuclides, especially that of ${ }^{68} \mathrm{Se}$ and ${ }^{64} \mathrm{Ge}$. Our mass measurements have shown that the rp-process path is delayed at ${ }^{64} \mathrm{Ge}$, and more significantly at ${ }^{68} \mathrm{Se}$. To date, the masses of more than 60 nuclides have been measured with the CPT, including nuclides along the rp-process path where mass information is needed the most, namely the proton-rich refractory metals and at the proposed termination of the rp-process path.

This work was supported by the U.S. Department of Energy, Office of Nuclear Physics, under Contract No. W-31-109-ENG38 , and by the Natural Sciences and Engineering Research Council of Canada.

\section{References}

1. J.A. Clark et al., in Exotic Nuclei and Atomic Masses (ENAM2001), Hämeenlinna, Finland, 2001, edited by J. Äystö, P. Dendooven, A. Jokinen, M. Leino (Springer, Berlin, 2003) p. 39.

2. J.A. Clark et al., Phys. Rev. Lett. 92, 192501 (2004).

3. G. Savard et al., Phys. Rev. C 70, 042501 (2004).

4. J.A. Clark et al., in The r-Process: The Astrophysical Origin of the Heavy Elements and Related Rare Isotope Accelerator Physics, Seattle, Washington, 2004, edited by Y.-Z. Qian, E. Rehm, H. Schatz, F.-K. Thielemann (World Scientific, Singapore, 2004) p. 11.

5. K.S. Sharma et al., these proceedings.

6. R.K. Wallace, S.E. Woosley, Astrophys. J. Suppl. Ser. 45 , 389 (1981).

7. H. Schatz et al., Phys. Rep. 294, 167 (1998).

8. M. Wiescher et al., J. Phys. G 25, R133 (1999).

9. T. Strohmayer, L. Bildsten, in Compact Stellar X-Ray Sources, edited by W.H.G. Lewin, M. van der Klis (Cambridge University Press, Cambridge) in press.

10. G. Savard et al., Nucl. Phys. A 626, 353 (1997).

11. J. Clark et al., Nucl. Instrum. Methods Phys. Res. B 204, 487 (2003).

12. G. Savard et al., Nucl. Instrum. Methods Phys. Res. B 204, 582 (2003).

13. C. Boudreau, Master's thesis, McGill University, 2001.

14. G. Savard et al., Phys. Lett. A 158, 247 (1991).

15. L.S. Brown, G. Gabrielse, Rev. Mod. Phys. 58, 233 (1986).

16. G. Bollen et al., J. Appl. Phys. 68, 4355 (1990).

17. M. König et al., Int. J. Mass Spectrom. Ion Processes 142, 95 (1995).

18. G. Bollen, Nucl. Phys. A 693, 3 (2001).

19. G. Gräff et al., Z. Phys. A 297, 35 (1980).

20. G.F. Lima et al., Phys. Rev. C 65, 044618 (2002).

21. M. Chartier, private communication.

22. A. Wöhr et al., Nucl. Phys. A 742, 349 (2004).

23. G. Audi et al., Nucl. Phys. A 729, 337 (2003).

24. B.A. Brown et al., Phys. Rev. C 65, 045802 (2002).

25. R. Pfaff et al., Phys. Rev. C 53, 1753 (1996).

26. J.A. Clark et al., in preparation.

27. H. Schatz et al., Phys. Rev. Lett. 86, 3471 (2001).

28. D.D. Clayton, F. Hoyle, Astrophys. J. 187, L101 (1974).

29. J. José et al., Astrophys. J. 520, 347 (1999).

30. J.C. Hardy et al., Phys. Rev. C 9, 252 (1974).

31. J.A. Nolen et al., Nucl. Instrum. Methods 115, 189 (1974).

32. S. Bishop et al., Phys. Rev. Lett. 90, 162501 (2003).

33. G. Audi, A.H. Wapstra, Nucl. Phys. A 595, 409 (1995).

34. J.C. Hardy et al., Phys. Rev. Lett. 91, 082501 (2003).

35. M. Mukherjee et al., Phys. Rev. Lett. 93, 150801 (2004).

36. D. Seweryniak et al., Phys. Rev. Lett. 94, 032501 (2005). 